\title{
Transglutaminase 2 ablation leads to mitophagy impairment associated with a metabolic shift towards aerobic glycolysis
}

\author{
F Rossin ${ }^{1,7}$, M D'Eletto ${ }^{1,7}$, L Falasca ${ }^{2}$, S Sepe ${ }^{3}$, S Cocco ${ }^{4}$, GM Fimia ${ }^{2,5}$, M Campanella ${ }^{4,6}$, PG Mastroberardino ${ }^{3}$, MG Farrace ${ }^{1}$ \\ and M Piacentini ${ }^{\star, 1,2}$
}

Macroautophagy selectively degrades dysfunctional mitochondria by a process known as mitophagy. Here we demonstrate the involvement of transglutaminase 2 (TG2) in the turnover and degradation of damaged mitochondria. In TG2-ablated cells we observed the presence of a large number of fragmented mitochondria that display decreased membrane potential, downregulation of IF1 along with increased Drp1 and PINK1 levels, two key proteins regulating the mitochondrial fission. Of note, we demonstrate that in healthy mitochondria, TG2 interacts with the dynamic proteins Drp1 and Fis1; interestingly, their interaction is largely reduced upon induction of the fission process by carbonyl cyanide $m$-chlorophenyl hydrazine (CCCP). In keeping with these findings, mitochondria lacking TG2 are more susceptible to CCCP treatment. As a consequence of accumulation of damaged mitochondria, cells lacking TG2 increased their aerobic glycolysis and became sensitive to the glycolytic inhibitor 2-deoxy-D-glucose (2-DG). In contrast, TG2-proficient cells are more resistant to 2-DG-induced apoptosis as the caspase 3 is inactivated through the enzyme's crosslinking activity. The data presented in this study show that TG2 plays a key role in cellular dynamics and consequently influences the energetic metabolism.

Cell Death and Differentiation (2015) 22, 408-418; doi:10.1038/cdd.2014.106; published online 25 July 2014

Autophagy is the cellular process responsible for the degradation of protein aggregates and dysfunctional organelles through the autophagosome-lysosome system. Under starvation conditions, bulk autophagy can be induced to catabolize cellular substrates to generate energy. However, it is now evident that autophagy is an on-going clearance mechanism for larger, longer-lived proteins and aggregates, as well as for organelles such as mitochondria and peroxisomes and pathogenic microorganisms. ${ }^{1}$ This basal cellular process takes places in all tissues contributing to the turnover of cytoplasmic components. In addition, autophagy is induced as an adaptive response to various physiological and pathological conditions. ${ }^{2}$ In fact, the proper regulation of the autophagic flux is fundamental for organism homeostasis under physiological conditions and even more so in response to metabolic stress, such as during physical activity and nutritional deficits. ${ }^{3}$

Autophagy plays an important role during cellular remodelling, in the course of embryonic development and ageing. ${ }^{4}$ Furthermore, its protective role in the major human diseases such as cancer, neurodegeneration and muscular disorders has been clearly demonstrated. . $^{5,6}$

Type 2 transglutaminase (TG2) is a member of a class of enzymes that catalyzes thiol- and calcium-dependent transamidation reactions. TG2 is a calcium-dependent enzyme and represents the most ubiquitous isoform, being expressed in many organs. Since the discovery of TG2 in $1957,{ }^{7}$ a large number of its substrates have been identified in intracellular compartments as well as on the cell surface and in the extracellular matrix. ${ }^{8,9}$ A peculiar aspect of TG2 biology is its multifunctionality, this being dictated by marked changes in the enzyme's 3-D structure. In fact, under physiological conditions the TG2 transamidating activities are inhibited by the binding of GTP, GDP and ATP that constrains the protein in the 'closed' conformation; ${ }^{10}$ in contrast, the transamidating activities are dependent on $\mathrm{Ca}^{2+}$ activation that shifts TG2 to the 'open' conformation, thereby unmasking the enzyme's active centre. The transamidase activity of TG2 leads to the incorporation/ deamidation of primary amines and of protein crosslinking by catalyzing the formation of isopeptide bond at the level of the amino groups of peptide-bound glutamine and lysine. ${ }^{11}$ In addition to its transamidase activity, TG2 has been shown to act as GTPase involved in the intracellular G protein signalling (as $G h \alpha$ ) at the level of adrenergic receptor. ${ }^{12}$ More recently, TG2 has also been reported to possess intracellular serine/threonine kinase and protein disulphide isomerase activity (PDI). ${ }^{13,14}$ The PDI enzymatic activity has been shown

${ }^{1}$ Department of Biology, University of Rome 'Tor Vergata', Rome, Italy; ${ }^{2}$ National Institute for Infectious Diseases I.R.C.C.S. 'L. Spallanzani', Rome, Italy; ${ }^{3}$ Department of Genetics, Erasmus Medical Center, Rotterdam, The Netherlands; ${ }^{4}$ European Brain Research Institute (EBRI), Rita Levi-Montalcini Foundation, Rome, Italy; ${ }^{5}$ Department of Biological and Environmental Sciences and Technologies (DiSTeBA), University of Salento, Lecce, Italy and ${ }^{6}$ Department of Comparative Biomedical Sciences, Royal Veterinary College, University of London and UCL Consortium for Mitochondrial Research (CfMR), London, UK

*Corresponding author: M Piacentini, Department of Biology, University of Rome 'Tor Vergata', Via della Ricerca Scientifica 00133, Rome, Italy. Tel: +39 06 72594234; Fax: +39 06 2023500; E-mail: mauro.piacentini@uniroma2.it

${ }^{7}$ These authors contributed equally to this work.

Abbreviations: 2-DG, 2-deoxy-D-glucose; BAP, 5-(biotinamido)pentylamine; CCCP, carbonyl cyanide m-chlorophenyl hydrazine; ECAR, extracellular acidification rate; HEK293, human embryonic kidney; MEF, mouse embryonic fibroblast; MMP, mitochondrial membrane potential; MTG, Mito Tracker Green; PHB, prohibitin; Strept, HRP-conjugated streptavidin; TG2, transglutaminase 2; TMRM, tetramethylrodhamine methyl ester; VCP, valosin-containing protein

Received 23.4.14; revised 11.6.14; accepted 13.6.14; Edited by G Kroemer; published online 25.7.14 
to lead to the post-translational modification of key mitochondrial proteins. ${ }^{15-19}$

Given the TG2 ubiquitous expression and variety of enzymatic and nonenzymatic activities, it is not surprising that this protein appears intimately involved in the regulation of numerous cell functions including cell adhesion, migration, survival and death, exocytosis and more recently autophagy. ${ }^{20}$ We have shown that the TG2 protein deletion, both in vivo and in mouse embryonic fibroblasts, results in the accumulation of LC3 II on preautophagic vesicles. ${ }^{21-23} \mathrm{We}$ have also demonstrated that TG2 regulation of autophagy occurs by its transamidating activity ${ }^{24}$ and its inhibition results in the intracellular increase of ubiquitinated protein aggregates. Interestingly, TG2 colocalizes in the protein complexes containing NBR1 and p62/SQSTM1, two adaptor proteins playing a key in the autophagic clearance of ubiquitinated proteins. $^{23}$

Considering all these findings and the evidence linking TG2 to mitochondria homeostasis, we decided to investigate TG2's role in autophagy regulation and organelles' quality control under stressful condition, focusing our studies on enzyme's impact on mitophagy and the aerobic metabolism.

\section{Results}

Several studies have proposed the involvement of TG2 in mitochondrial homeostasis. Indeed, it has been clearly shown that TG2 is implicated in the homeostasis of the mitochondrial respiratory chain. ${ }^{13,25}$ In keeping with this notion, some of the characterized TG2 substrates (Prohibitin, ATP synthase $\beta$, ANT-1) play a key role in the mitochondrial homeostasis. ${ }^{26,14}$ Prompted by such evidence, we decided to study a possible role of the enzyme in mitochondrial homeostasis, with particular regard to removal of dysfunctional mitochondria by autophagy and its impact on the overall cellular metabolism. To this end, we used MEFs obtained from wild-type (WT MEFs) and TG2 knockout (KO MEFs) mice and HEK293 cells stably transfected with the WT enzyme (HEK293 $\left.{ }^{\mathrm{TG} 2}\right)^{23}$ that constitutively express very low level of TG2 (Supplementary Figures S1A and B).

\section{Effect of TG2 ablation on mitochondrial morphology and} function. In order to define the role of TG2 in mitochondrial physiology, we first performed morphological analyses of these organelles in the presence and absence of TG2. We started analysing the mitochondrial network by fluorescent microscopy carried out in WT MEFs and KO MEFs under basal conditions. Figure 1a shows representative images demonstrating the presence of a significant amount of fragmented mitochondria in cells lacking TG2 compared with the tubular ones observed in WT MEFs. We quantified this variance by counting the percentage of cells showing fragmented mitochondria, as reported in Figure $1 \mathrm{~b}$. In keeping with this finding, transmission electron microscopy reveals that KO MEFs display damaged swollen mitochondria with altered morphology and aberrant inner-membrane a

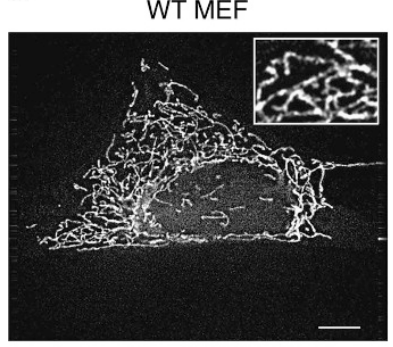

C

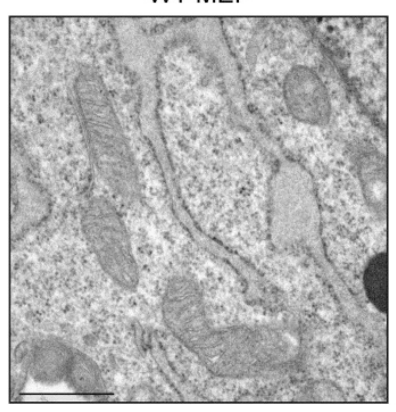

KO MEF

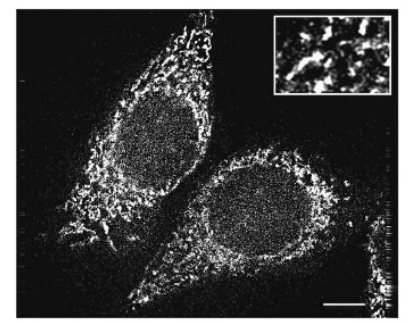

KO MEF

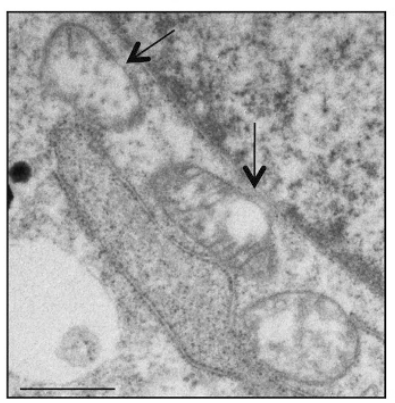

b

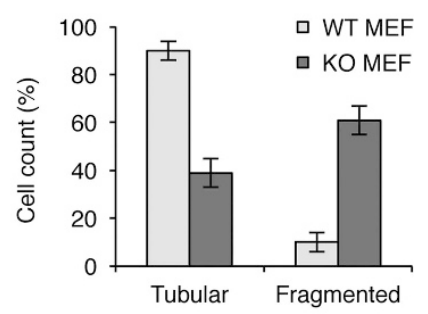

d

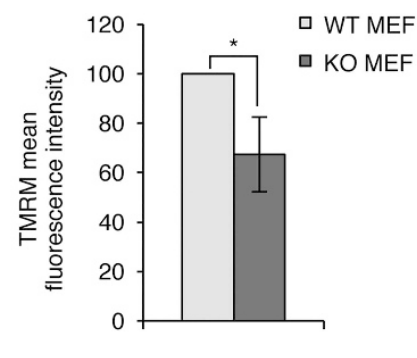

Figure 1 Loss of TG2 causes alterations in mitochondrial morphology and functionality. (a) Fluorescence microscopy analysis in WT and KO MEF cell lines under basal conditions. Cells were transiently transfected with the mitochondrial marker pDsRed2-Mito (red fluorescence) and analysed by microscopy. Magnified images are shown in the insets. Scale bar, $6 \mu \mathrm{m}$. (b) Quantitative analysis of the mitochondrial morphology. The number of cells containing fragmented and tubular mitochondria was expressed as percentage of total counted cells. The cells were counted in five independent fields from three independent experiments. Results are expressed as mean \pm S.D. (c) Transmission electron microscopy in WT and KO MEFs under basal conditions. Mitochondria with altered morphology and inner-membrane cristae are detected in KO MEF cells (arrows). Scale bar, $1 \mu \mathrm{m}$. (d) Mitochondria $\Delta \psi \mathrm{m}$ was determined by TMRM staining and FACS analysis. WT and KO MEFs were harvested and stained with TMRM for 20 min. Quantification of TMRM mean fluorescence intensity in MEF cell lines is referred as percentage of WT value. Results are expressed as mean \pm S.D. of three independent experiments $\left({ }^{*} P<0.05\right)$ 
cristae (Figure 1c). To define whether these morphological defects were correlated to mitochondrial dysfunctions, we decided to examine mitochondrial functionality measuring mitochondrial membrane potential. To this end, we performed a cytofluorimetric analysis in WT and KO MEFs treated with the lipophilic cationic dye TMRM, a membrane potential-dependent marker accumulating in functional mitochondria. The data reported in Figure 1d evidenced a decrease in the TMRM red-orange fluorescence signal in the cells lacking TG2, indicating the depolarization of membrane in dysfunctional mitochondria.

Lack of TG2 leads to an impaired mitophagy. Considering that cells lacking TG2 display dysfunctional mitochondria, we decided to study whether TG2 could be involved in the mitochondrial response to damage. We therefore treated WT and KO MEFs as well as HEK293 and HEK293 ${ }^{\text {TG2 }}$ with the uncoupler carbonyl cyanide m-chlorophenyl hydrazine (CCCP) for $18 \mathrm{~h}$ to induce mitochondrial damage and eventually the clearance by mitophagy. The ultrastructural analysis of both cell lines upon $18 \mathrm{~h}$ of treatment with CCCP revealed profound difference in the morphology comparing cells lacking TG2 (KO MEF and HEK293) and cells with the enzyme. In the latter, the cytoplasm shows a drastic accumulation of damaged mitochondria and autophagosomes containing undigested materials (Supplementary Figures S2 and S3). In keeping with this finding, we quantified by flow cytometry analysis the relative mitochondrial mass by Mito Tracker Green (MTG) staining. ${ }^{27}$ As shown in Figure 2a, upon $24 \mathrm{~h}$ of damage induction, $\mathrm{KO}$ MEFs exhibit a significant increase in mitochondrial mass compared with WT cells, suggesting a possible impairment of mitophagy. This finding is also supported by the differential accumulation of PTEN-induced putative kinase 1 (PINK1) that is a key element of mitochondrial quality control specifically targeting these organelles for degradation. In fact, upon CCCP treatment we observed the accumulation of PINK1 in KO MEFs supporting an impairment of the mitophagic process (Figure $2 b$ ). Similar results were obtained in HEK293 cells (Supplementary Figures S1C and D), confirming the negative effect of the ablation of TG2 on mitochondrial clearance. In keeping with these findings, we also detected the selective degradation of mitofusins, essential proteins in the regulation of mitochondrial dynamics, ${ }^{28,29}$ exclusively in MEFs from WT mice upon CCCP treatment. In fact, no mitofusin degradation was observed in MEFs from $\mathrm{KO}$ animals (Figure 2c). Interestingly, the mitofusin degradation can be prevented by blocking the lysosomal/autophagic activity by $\mathrm{NH}_{4} \mathrm{Cl}$ in MEFs from WT animals, further indicating the impairment of mitophagy in cells lacking TG2. To further support this hypothesis, we examined the degradation of mitochondria by analysing their colocalization with lysosomes. The immunofluorescence analysis carried out in WT and KO MEFs (Figure 2d) clearly indicates that cells lacking TG2 have impaired clearance of defective mitochondria, as highlighted by the absence of overlay between Lyso Tracker Red (lysosomes) and Tom20 (mitochondria).

To get insights into TG2 involvement in the process, we monitored TG2 transamidating activity by detecting a

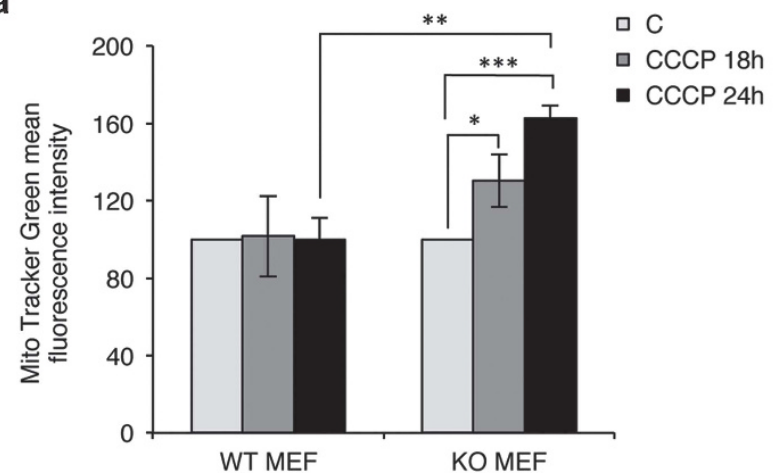

b

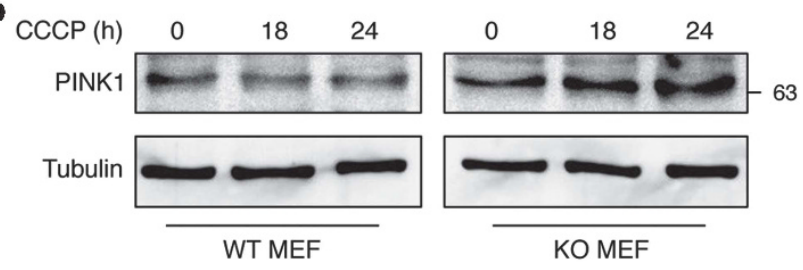

C

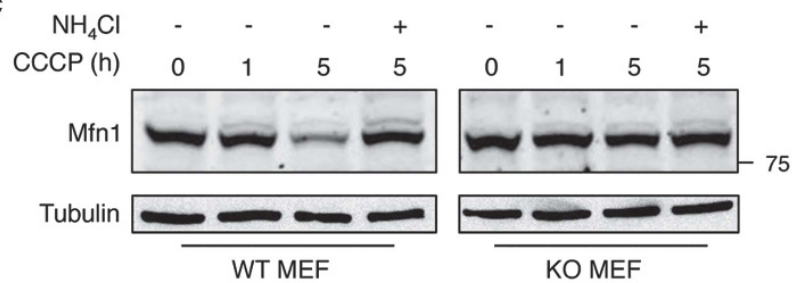

d

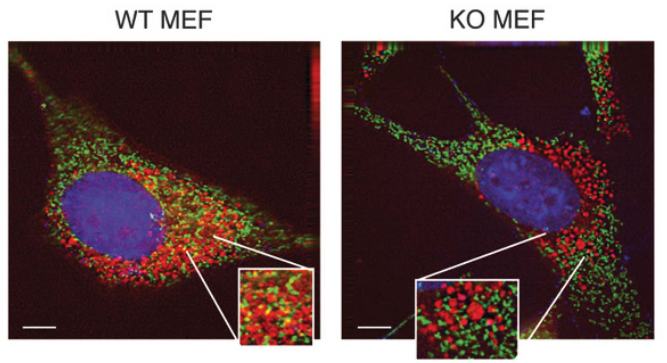

Figure 2 Lack of TG2 leads to an impaired mitophagy. (a) Mitochondrial mass was evaluated in WT and KO MEFs by Mito Tracker Green (MTG) staining and quantified by FACS. Cells were treated with CCCP for 18 and $24 \mathrm{~h}$, harvested and stained with MTG for $30 \mathrm{~min}$. Quantification of MTG mean fluorescence intensity in MEF cell lines is referred as percentage of control value. Results are expressed as mean \pm S.D. of three independent experiments $\left({ }^{\star} P<0.05, \quad{ }^{\star \star} P<0.001\right.$, $\left.{ }^{* * *} P<0.0001\right)$. (b) Representative western blot analysis of the mitochondrial protein PINK1 in WT and KO MEFs upon CCCP treatment for 18 and $24 \mathrm{~h}$. Tubulin was used as loading control $(n=3)$. (c) Representative western blot analysis of the mitochondrial protein mitofusin 1 (Mfn1) in WT and KO MEFs upon CCCP treatment for 1 and $5 \mathrm{~h}$. $\mathrm{NH}_{4} \mathrm{Cl}$ was used for $5 \mathrm{~h}$ to inhibit the activation of the lysosomal enzymes blocking the degradation process. Tubulin was used as loading control $(n=3)$. (d) Immunofluorescence microscopy analysis of MEF cells after CCCP treatment for $24 \mathrm{~h}$. Lyso Tracker Red (red); Hoechst (blue); Tom20 (green); merge (yellow). Green dots represent mitochondria, red dots represent free lysosomes and yellow dots are sites of overlap. Magnified images are shown in the insets. Scale bar, $6 \mu \mathrm{m}$

5-(biotinamido)pentylamine (BAP) incorporation into mitochondrial proteins upon CCCP treatment. Figure 3a clearly indicates a drastic increase of the enzyme's transamidating 
a

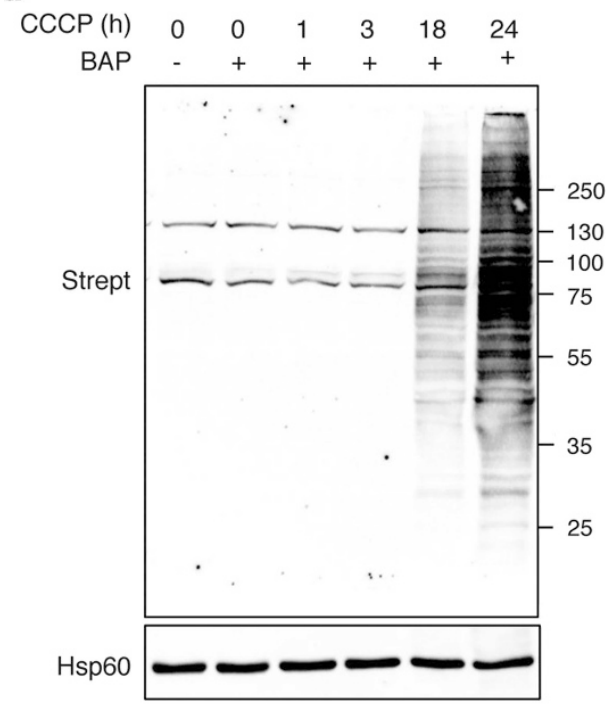

b

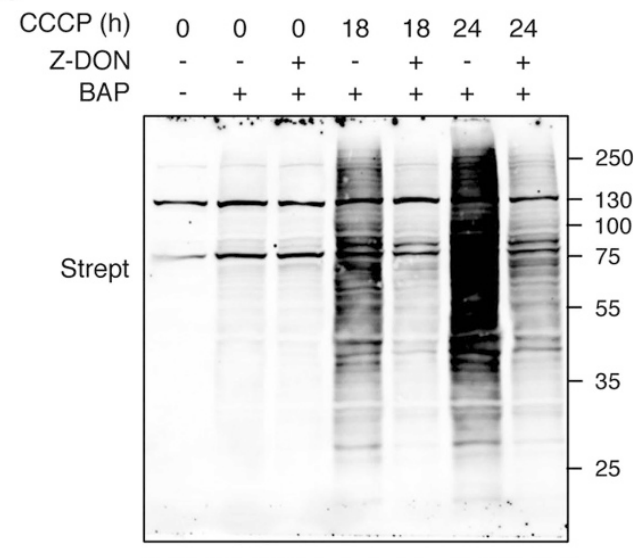

Hsp60

C

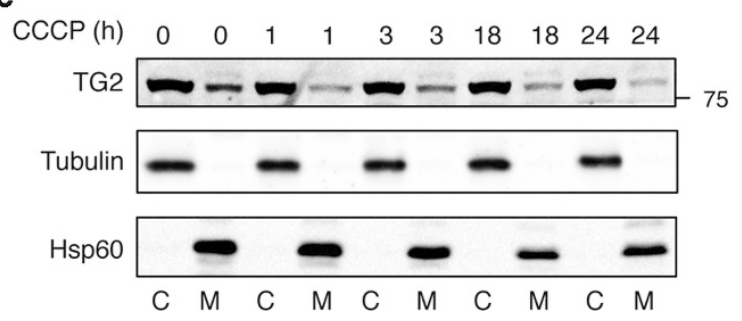

Figure 3 Analysis of TG2 transamidating activity and protein expression levels. (a and $\mathbf{b}$ ) Representative blot of the TG-catalyzed incorporation of 5-(biotinamido)pentylamine (BAP) into mitochondrial proteins in WT MEFs as a measure of in situ TG2 activity. Cells were labelled with BAP and treated with CCCP and/or Z-DON. After subcellular fractionation, mitochondrial proteins were separated by SDS-PAGE and biotinylated proteins were revealed with HRP-conjugated streptavidin (strept). Hsp60 was used as loading control $(n=3)$. (c) Cytosolic and mitochondrial fractions from WT MEFs after mitochondrial damage induction by using CCCP. Fractions were subjected to immunoblotting for the expression of TG2. Hsp60 and tubulin were used as loading control respectively for mitochondrial and cytoplasmic fractions $(n=3)$

activity on mitochondria that is evident after $18 \mathrm{~h}$ of CCCP treatment, in coincidence with the onset of mitophagy. ${ }^{30}$ In line with this finding, it has been shown that the mitophagy onset is paralleled by the release of free calcium from the endoplasmic reticulum that is essential for TG2 activation. ${ }^{31,32}$ It is important to note that the enzyme's activation on mitochondria is organelle specific as the same analysis carried out on the cytosolic fraction shows a very limited transamidating activity (Supplementary Figure S4A). To exclude the possibility that the transamidating activity was because of other transglutaminases, we also treated the MEFs with Z-DON, a highly specific TG2 inhibitor (Figure 3b). Interestingly, the incorporation of BAP was largely inhibited by Z-DON, thus indicating that the observed transamidating activity was because of the activation of TG2. It is interesting to note that the TG2 protein levels on mitochondria show a progressive decrease after CCCP treatment (Figure 3c). A similar trend was also observed in HEK293 ${ }^{\mathrm{TG} 2}$ cells expressing high levels of TG2 (Supplementary Figure S4B).

To define at the molecular level the presence of damaged mitochondria in the absence of TG2, we evaluated the accumulation of the Drp1 protein on mitochondria. Drp1 is a cytosolic protein recruited to mitochondria to carry out their fragmentation and thus facilitate their clearance by autophagy. ${ }^{33}$ Interestingly, already in untreated MEFs from KO mice, we detected an enhancement of Drp1 levels (threefold higher, $P<0.001$; Supplementary Figure S4C) on mitochondria, thus indicating their impairment and priming to undergo fission. Upon CCCP damage, Drp1 translocates on mitochondria in both WT and KO MEFs (Figures $4 \mathrm{a}$ and b); however, in WT cells the protein levels go back to the original level at later time points that coincide with mitophagy induction. In contrast, the Drp1 level on the mitochondria from KO MEFs keeps increasing, thus indicating the presence of undegraded damaged mitochondria. To further evaluate the role of TG2 in the clearance of mitochondria, we analysed the TG2 enzymatic activity upon mitochondrial damage induction in the presence of Mdivi-1, an indirect inhibitor of mitophagy. ${ }^{34}$ Interestingly, the increased TG2-dependent post-translational modification of substrate proteins observed at later times of CCCP treatment is largely reduced in the presence of Mdivi-1 (Figure 4c), thus further indicating the involvement of the enzyme in the regulation of mitophagy.

Fission is a prerequisite for mitophagy to occur, but when this protective mechanism fails, mitochondrial fission can also promote apoptosis. Indeed, impairment of mitophagy leads to the accumulation of dysfunctional mitochondria and makes the cells more susceptible to MMP and cell death. ${ }^{35}$ It has 
a

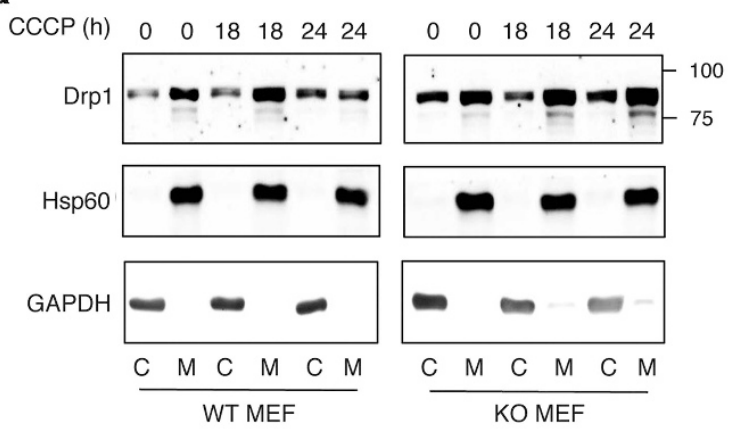

b

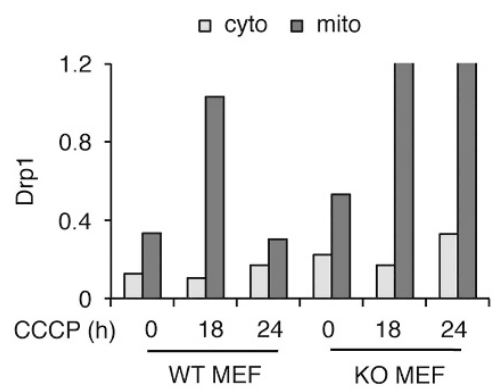

C

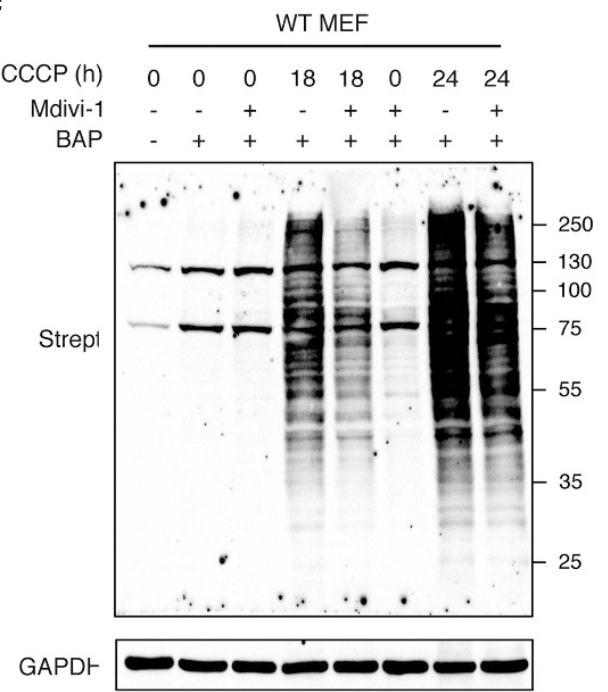

Figure 4 TG2 transamidating activity is involved in the removal of fragmented mitochondria by mitophagy. (a) Representative blot of cytosolic and mitochondrial fractions from WT and KO MEFs after mitochondrial damage induction by using CCCP. Fractions were subjected to immunoblotting for the expression of Drp1. Hsp60 and GAPDH were used as loading control respectively for mitochondrial and cytoplasmic fractions $(n=3)$. (b) Densitometric analysis of the represented western blot (quantification of cytosolic and mitochondrial Drp1 bands respectively normalized to GAPDH and Hsp60 levels). (c) Representative blot of the TG-catalyzed incorporation of 5-(biotinamido)pentylamine (BAP) into proteins in WT MEFs as a measure of in situ TG2 activity. Cells were labelled with BAP and treated with CCCP in the presence or not of Mdivi-1. After separation by SDS-PAGE, biotinylated proteins were revealed with HRP-conjugated streptavidin (strept). GAPDH was used as loading control ( $n=3$ )

previously been shown that IF1 protects cells from both ischemic and apoptotic type of cell death respectively by (1) limiting the reversion of the F1Fo-ATPsynthase during blockage of cell respiration and (2) supporting cristae structure via the dimerization of the $\mathrm{F} \mathrm{F}_{0}$-ATP synthase. ${ }^{36,37}$ In fact, IF1 plays a key role in the preservation of mitochondrial morphology and cristae architecture, supporting its role in the modulation of cytochrome $c$ release and activation of the apoptosis via the intrinsic pathway. ${ }^{37}$ In addition, IF1 has been identified as an essential factor for PARK2 recruitment and consequently mitophagy activation. ${ }^{38}$ In accordance with the above described protective effect of TG2 on mitochondria, we detected a drastic reduction of IF1 protein level in TG2-null MEFs, untreated cells. Interestingly, we observed a very different IF1 protein turnover in the presence and absence of TG2 that is independent by autophagy (Figure 5a). However, the lack of TG2 in KO MEF correlates with the functional analysis of $\mathrm{F}_{1} \mathrm{~F}_{0}$-ATP synthase that is indeed acting in reverse (Figures $5 \mathrm{a}$ and $\mathrm{b}$ ). In fact, the reversion of $\mathrm{F}_{1 \mathrm{~F}_{0}-\mathrm{ATP}}$ synthase, shown in Figure 5b, is unmasked by oligomycin that, by dropping the $\Delta \psi_{\mathrm{m}}$, highlights an inverse way of rotation of the enzyme. These data further indicate the mitochondrial dysfunction priming these cells for cell death induction. To this aim we analysed apoptosis induction in the absence of TG2. As expected, upon $24 \mathrm{~h}$ of CCCP treatment, caspase 3 is activated in KO MEFs but not in WT ones (Figure 5c). Interestingly, the cleavage of caspase 3 can be observed in WT cells only after the inhibition of autophagy by $\mathrm{NH}_{4} \mathrm{Cl}$ that prevents the clearance of damaged mitochondria. In keeping with these findings, we also detected the translocation of GAPDH on mitochondria only in MEFs lacking TG2 (Figure $5 \mathrm{~d}$ ). It has been shown that under stressful cellular conditions, GAPDH interacts with the voltage-dependent anion channel (VDAC), promoting the cytochrome $c$ and apoptosis-inducing factor release, leading to apoptotic cell death. ${ }^{39}$

TG2 interacts with key elements of the fission/fusion molecular pathway. In order to define the molecular mechanisms at the basis of above described effects of TG2 on mitophagy, we analysed the interaction of the enzyme with the mitochondrial Drp1 and Fis1, two key 
a

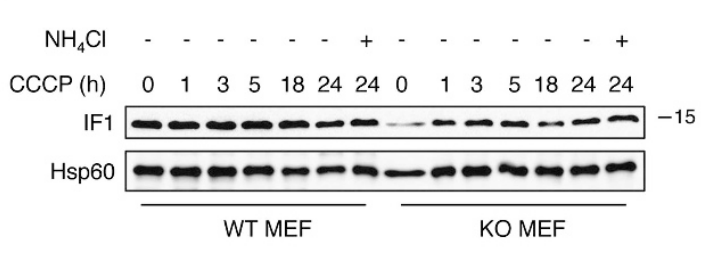

b

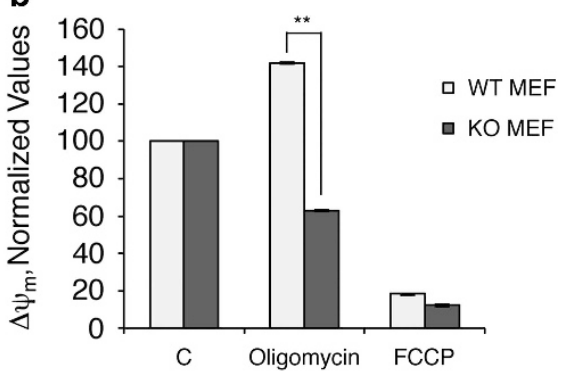

c

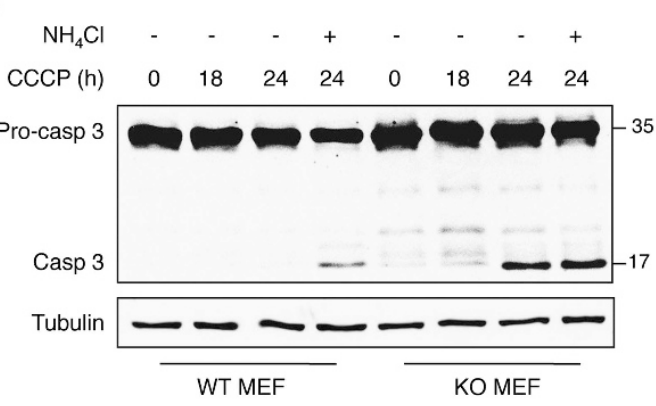

d
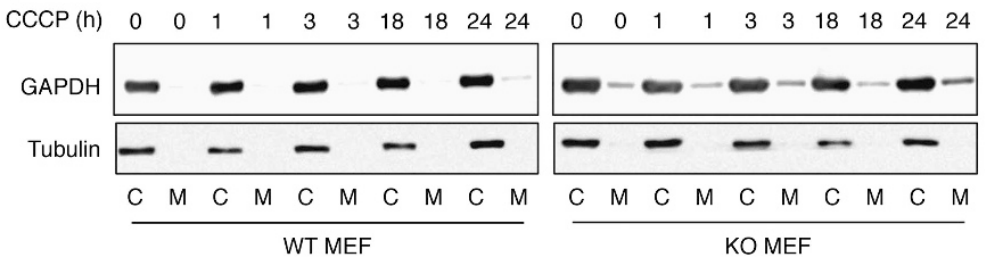

Figure 5 Mitophagy impairment leads to caspase 3 activation in TG2 KO cells. (a) Representative western blot of IF1 protein in the mitochondrial fraction of WT and KO MEFs upon CCCP treatment. Hsp60 was used as loading control $(n=3)$. (b) Data points of $\Delta \psi \mathrm{m}$ in WT and KO MEFs in response to the pharmacological inhibition of the

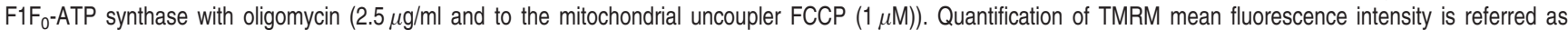
percentage of control value. Results are expressed as mean \pm S.D. of three independent experiments $\left({ }^{\star \star} P<0.001\right)$. (c) Representative western blot analysis of caspase 3 activation in WT and KO MEFs upon CCCP treatment for 18 and $24 \mathrm{~h}$ in the presence or not of $\mathrm{NH}_{4} \mathrm{Cl}$ to inhibit the activation of the lysosomal enzymes blocking the degradation process. Tubulin was used as loading control $(n=3)$. (d) Cytosolic and mitochondrial fractions from WT and KO MEFs after mitochondrial damage induction by using CCCP. Representative western blot shows the expression of GAPDH and tubulin $(n=3)$

proteins regulating mitochondrial fission. To this end we used HEK293 cells reconstituted with TG2 as HEK293 express a very low amount of enzyme (Supplementary Figure S1B).

Data reported in Figures $6 a$ and c clearly show that TG2 constitutively interacts with both Drp1 and Fis1 at the mitochondrial level (Figures 6a and c, respectively). However, upon CCCP treatment, the interaction of TG2 with Fis1 and particularly Drp1 shows a drastic reduction $(\sim 50 \%)$ at the mitochondrial level (Figures $6 \mathrm{~b}$ and $\mathrm{d}$ ). Interestingly, upon CCCP treatment, TG2 level is rapidly and significantly reduced on the mitochondria (Figure $3 \mathrm{c}$ ), thus very likely favouring the interaction between Drp1 with its receptor Fis1 leading to mitochondria fission. Of note, TG2 interacts with Drp1 in the cytoplasm and this interaction is not significantly modified by the CCCP treatment (Figure 6a). Of note, in the same protein complex we detected the presence of prohibitin (PHB) that displayed an increased interaction with TG2 upon CCCP treatment (Supplementary Figure S4D). PHB is a well-known TG2 substrate that protects cells from mitophagy. ${ }^{26,40}$ Finally, we observed an early significant reduction in valosin-containing protein (VCP) levels in the mitochondria of HEK293 ${ }^{\text {TG2 }}$ upon CCCP treatment that was not displayed in HEK293 cells lacking TG2 expression (Figures $6 \mathrm{e}$ and f). Interestingly, VCP is recruited to damaged mitochondria being involved in their clearance via the PINK1/Parkin pathway. ${ }^{41}$ These findings indicate that TG2 might play an important role in mitophagy regulation by its direct interaction with key components of the fission/fusion molecular machinery.

TG2 KO cells show alteration of the aerobic metabolism. Considering that the absence of TG2 causes alterations in the mitochondria functionality, particularly evident under stressful conditions, we asked how TG2 deletion affects the overall cell metabolism. We therefore measured the extracellular acidification rate (ECAR), as an indicator of the glycolytic flux, in primary MEFs from WT and TG2 KO mice. As shown in Figures 7a and b, lack of TG2 results in higher glycolytic activity that in turn causes increased acidification of the culture medium. In order to further define this finding, we analysed essential regulators of the glycolytic pathway that are also known interactors or substrates of TG2, for instance, 
a

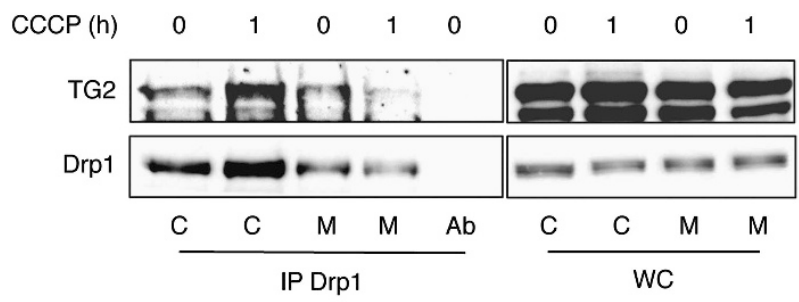

C

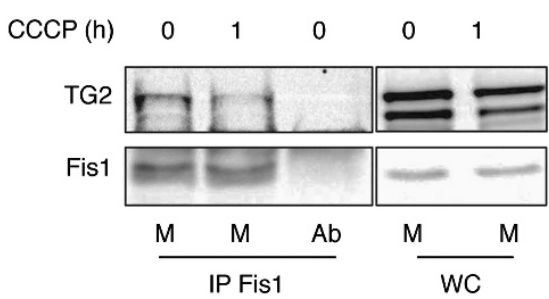

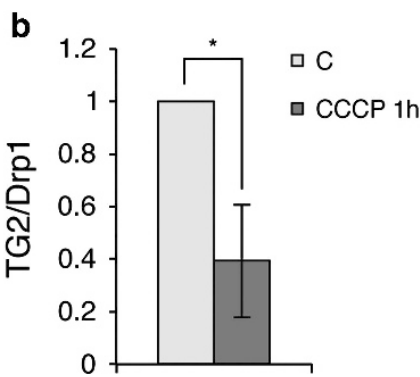

d

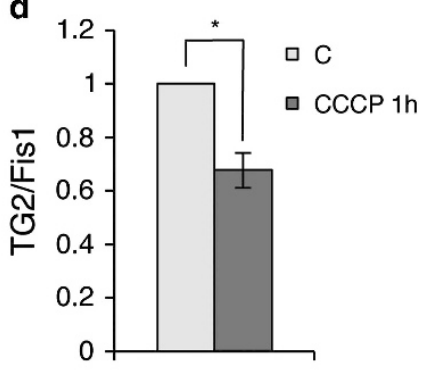

e
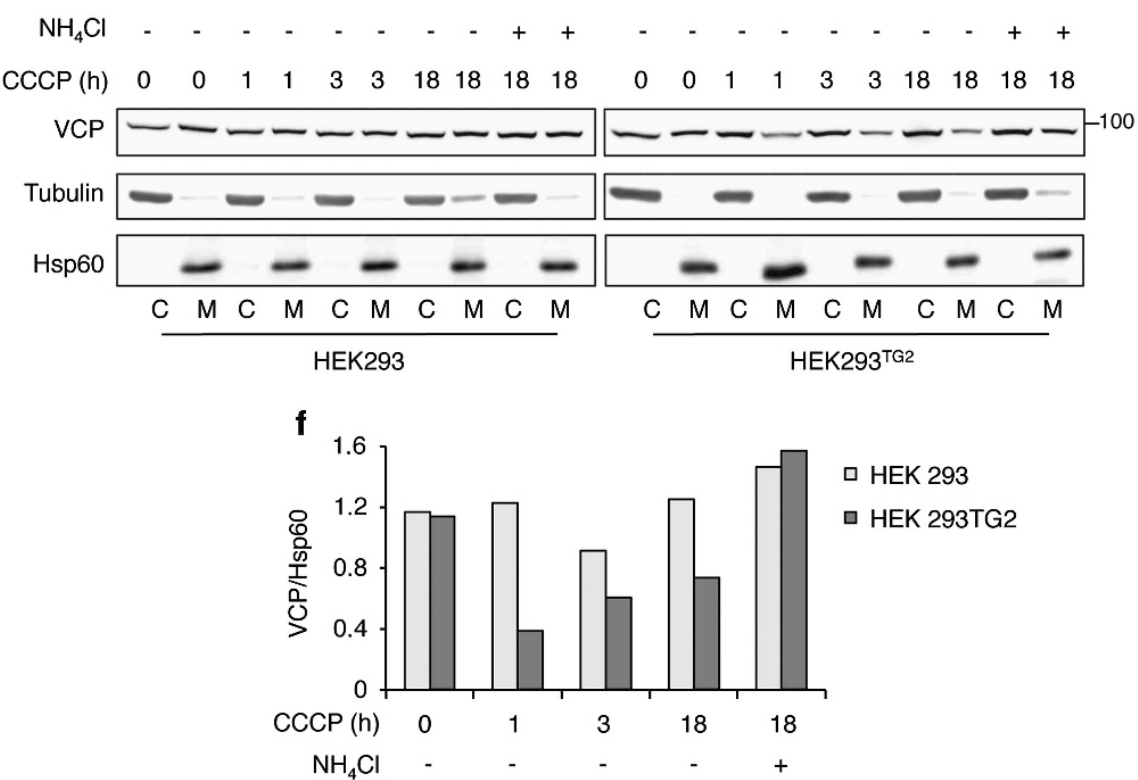

口 HEK 293

口 HEK 293TG2

Figure 6 TG2 interacts with Drp1 and Fis1. (a) Representative western blot analysis of TG2 and Drp1 in HEK293 ${ }^{\text {TG2 }}$ cells subjected to immunoprecipitation for Drp1. After $1 \mathrm{~h}$ of CCCP treatment, cells were lysed, cytosolic and mitochondrial proteins were immunoprecipitated using anti-Drp1 antibody (see Materials and Methods). Immuno- and co-immunoprecipitated proteins were separated by SDS-PAGE and immunoblotted using the indicated antibodies. Whole cell lysate (WC) was used as protein control ( $n=3)$. (b) Densitometric analysis of the ratio of co-immunoprecipitated TG2/Drp1 in the mitochondrial fraction, referred as percentage of control values. Results are expressed as mean \pm S.D. of three independent experiments $\left({ }^{*} P<0.05\right)$. (c) Representative western blot analysis of TG2 and Fis1 in mitochondria from HEK293 ${ }^{\text {TG2 }}$ cells subjected to immunoprecipitation for Fis1 after $1 \mathrm{~h}$ of CCCP treatment, as previously reported. Immuno- and co-immunoprecipitated proteins were immunoblotted using the indicated antibodies. WC was used as protein control $(n=3)$. (d) Densitometric analysis of the ratio of co-immunoprecipitated TG2/Fis1 in the mitochondrial fraction, referred as percentage of control values. Results are expressed as mean \pm S.D. of three independent experiments $\left({ }^{*} P<0.05\right)$. (e) Cytosolic and mitochondrial fractions from HEK293 and HEK293 ${ }^{\text {TG2 }}$ cells after mitochondrial damage induction by using CCCP in the presence or not of $\mathrm{NH}_{4} \mathrm{Cl}$ used to inhibit the activation of the lysosomal enzymes blocking the degradation process. Fractions were subjected to immunoblotting for the expression of VCP. Hsp60 and tubulin were used as loading control respectively for mitochondrial and cytoplasmic fractions $(n=3)$. (f) Densitometric analysis of the western blot (quantification of mitochondrial VCP bands normalized to Hsp60 levels)

GAPDH and HIF-1 $\beta .^{42,43}$ The western blots analyses displayed in Figure 7c show that, in the absence of TG2, there is an upregulation of both GAPDH and HIF-1 $\beta$, thus supporting the observed induction of glycolysis in cells lacking TG2. Consistently, the glycolysis inhibition by 2-deoxy-D-glucose (2-DG), a structural analogue of glucose, leads to a marked activation of transglutaminase activity as evidenced by the incorporation of BAP into cellular proteins (Figure 7d). Interestingly, the increased TG2 activity leads to caspase 3 crosslinking and consequent inhibition of its proapoptotic activity. Crosslinking of caspase 3 is mediated by TG2 rather than other transglutaminases because it cannot 
a

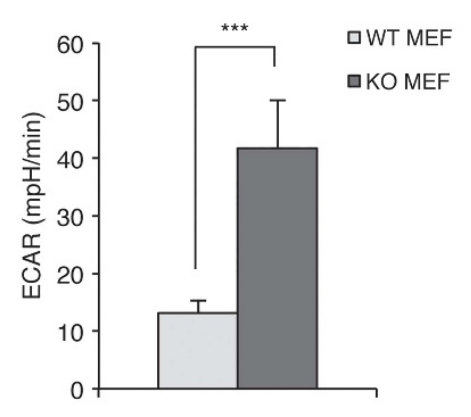

b

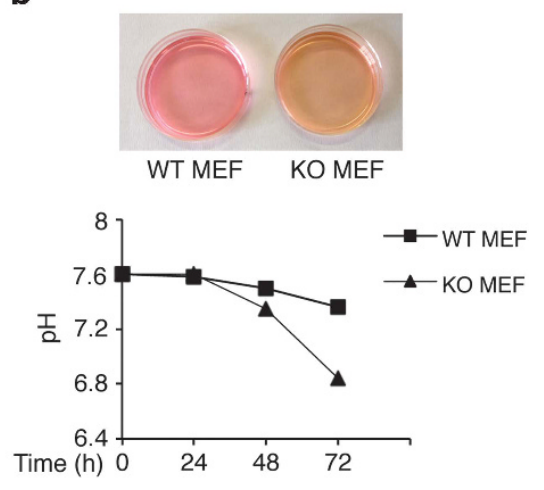

d

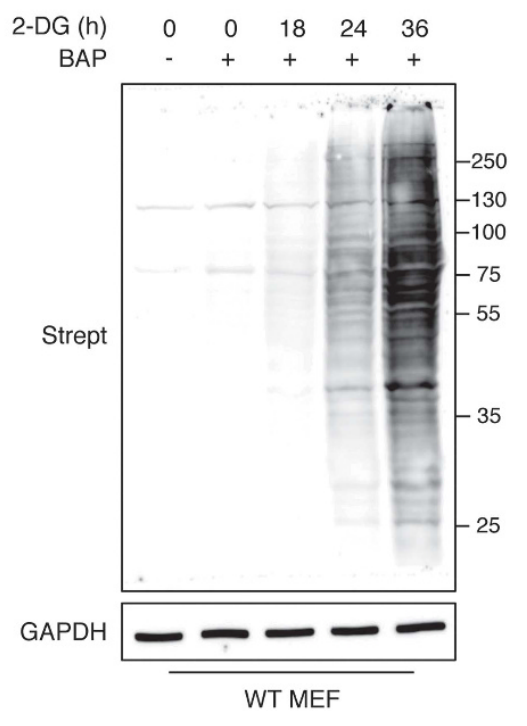

$\begin{array}{llllllllllll}\text { e } & 2-D G(h) & 0 & 6 & 18 & 24 & 36 & 0 & 6 & 18 & 24 & 36\end{array}$

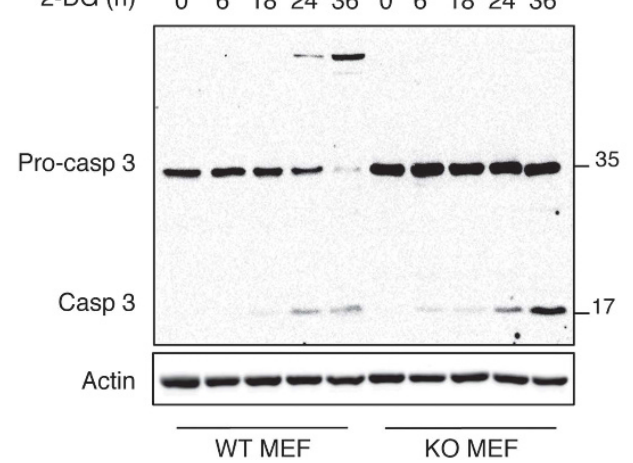$$
\text { WT MEF KOMEF }
$$

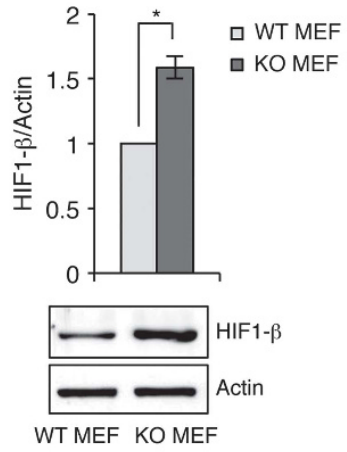

WT MEF KO MEF

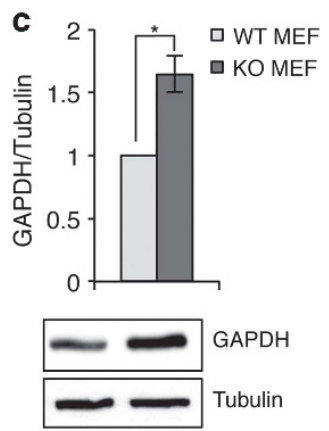

WT MEF KO MEF

Figure 7 TG2 KO cells display a shift to aerobic glycolysis. (a) Analysis of extracellular acidification rate (ECAR) measured in primary WT and KO MEFs, using the Seahorse XF technology, to establish baseline rate. Results are expressed as mean \pm S.D. of three independent experiments $\left.{ }^{* * *} P<0.0001\right)$. (b, upper panel) Representative experiment showing cell medium acidification after 6 days of culture in WT and KO MEF cells. (b, lower panel) pH determination at different points in WT and KO MEFs. (c, left) Densitometric analysis of GAPDH blots normalized to tubulin levels, referred as percentage of WT values. Representative western blot of GAPDH in WT and KO MEF cells. (c, right) Densitometric analysis of HIF- $1 \beta$ blots normalized to actin levels, referred as percentage of WT values. Representative western blot of HIF- $\beta$ in WT and KO MEFs. Results are expressed as mean \pm S.D. of three independent experiments $\left({ }^{*} P<0.05\right)$. (d) Representative blot of the TG-catalyzed incorporation of 5-(biotinamido)pentylamine (BAP) into proteins in WT MEFs as a measure of in situ TG2 activity. Cells were labelled with BAP and treated with 2-DG as indicated. After separation by SDS-PAGE, biotinylated proteins were revealed with HRP-conjugated streptavidin (strept). GAPDH was used as loading control ( $n=3$ ). (e) Western blot analysis of caspase 3 activation in WT and KO MEFs upon 2-DG treatment. Actin was used as loading control $(n=3)$

be detected in KO MEFs (Figure 7e), exhibiting a clear caspase 3 activation.

\section{Discussion}

It is well documented that autophagy plays an essential role in the cellular organelle turnover, selectively degrading dysfunctional or supernumerary mitochondria by a process known as mitophagy. This study provides that TG2 is involved in mitophagy particularly under stressful cellular conditions. It has been previously shown that the TG2 transamidating activity inhibition resulted in an evident accumulation of autophagosomes, suggesting the impairment in the final stages of the autophagy process. ${ }^{22-24}$ In this work, we demonstrate a TG2-protective role in the turnover and degradation of dysfunctional mitochondria. In fact, in the absence of the enzyme we observed an accumulation of dysfunctional mitochondria in two different cell lines, thus 
suggesting a role for TG2 in the regulation of mitochondrial homeostasis. Furthermore, we show a mitochondria-specific activation of the enzyme transamidating activity upon mitochondrial damage (CCCP treatment). We demonstrated that cells lacking TG2 display altered mitochondrial morphology and functionality, as shown by the presence of more fragmented and depolarized mitochondria, paralleled by a drastic suppression of IF1, a molecule that associates with the preservation of mitochondrial morphology, ultrastructure and the efficiency of the mitophagy cascade. The IF1 reduced expression links with the functional analysis of the F1FoATPsynthase that is indeed acting in reverse, an adaptation that may well fit with the metabolic phenotype of the TG2 KO cells in which the glycolytic metabolism results increased. These results are in keeping with a previous study demonstrating that deletion of TG2 in mice caused a significant deregulation of the respiratory complexes I and II and a reduction of ATP production and confirm a role for the enzyme as a protective element in the regulation of mitochondrial homeostasis and metabolism. ${ }^{25}$ In line with this notion, cells knockout for TG2 displayed, before and after damage, a sustained higher mitochondrial level of the fission protein Drp1, a primary regulator of mitochondrial fission. ${ }^{44}$ Interestingly, we observed the interaction of endogenous TG2 with Drp1 in both the cytosol and at mitochondrial level; this physical interaction, however, significantly decreased after a sustained mitochondrial damage as well as the interaction of TG2 with Fis1. Based on these findings, it is tempting to hypothesize that TG2 interferes with Drp1 binding to its mitochondrial receptors, therefore delaying mitochondrial fission preceding mitophagy (Figure 8). Of note, we showed that Mdivi-1, a cell-permeable inhibitor of Drp1-mediated mitochondrial fission, leads to a marker decrease of TG2 intracellular crosslinking activity that coincides with the onset of mitophagy. ${ }^{45,46}$ This compound, by blocking the GTPase activity, inhibits the oligomerization of Drp1, thus preventing the mitochondrial fission. ${ }^{47}$ Interestingly, this compound has been proposed as a potential new drug for neurodegenerative diseases. ${ }^{48,49}$ To this aim, it is relevant that TG2 has been shown to play a role in the pathogenesis of neurodegenerative diseases characterized by mitochondria impairment, such as Parkinson's disease and Huntington's disease. ${ }^{17,50}$ Thus, future studies should clarify by which mechanism Mdivi-1 inhibits TG2 transamidating activity and whether this might represent a possibility to treat neurodegenerative disease.

We also demonstrated that, in order to survive, cells lacking TG2 switch to a higher rate of aerobic glycolysis and are more sensitive to the glycolytic inhibitor 2-DG. Overall, these metabolic rearrangements indicate a compensatory alteration of the mitochondrial metabolism in the absence of the enzyme, suggesting a role for TG2 in the regulation of the switch of cellular metabolism from mitochondrial respiration to aerobic glycolysis. Finally, we observed that the accumulation of fragmented mitochondria, in the absence of TG2, leads to caspase 3 activation. According to this result, WT cells undergo apoptosis only when the degradation of mitochondria by autophagy is inhibited. In this regard, it is known that fission facilitates to remove damaged mitochondria, leading to their degradation by autophagy; however, when this protective mechanism is defective, mitochondrial fission can promote apoptosis as well. ${ }^{35}$ In line with the herein proposed protective role displayed by TG2 upon mitochondrial damage, we demonstrated that the transamidating activity of TG2 leads to inhibition/delay of apoptosis by crosslinking caspase 3, thus impairing its activation. It is important to note that the transamidating activity of TG2 increased at later times after CCCP treatment as a specific protective event associated with the accumulation of dysfunctional mitochondria, as confirmed by the absence of transamidating activity in cells treated with the mitophagy inhibitor Mdivi-1. In keeping with this, it is accepted that upon mitophagy induction there is an increase of free calcium ions in the cytoplasm that may result in the activation of the transamidating activity of TG2. ${ }^{31,32,51}$ Overall, these findings highlight the essential protective role played by TG2 in the regulation of mitochondria functionality as well as in their clearance by mitophagy.

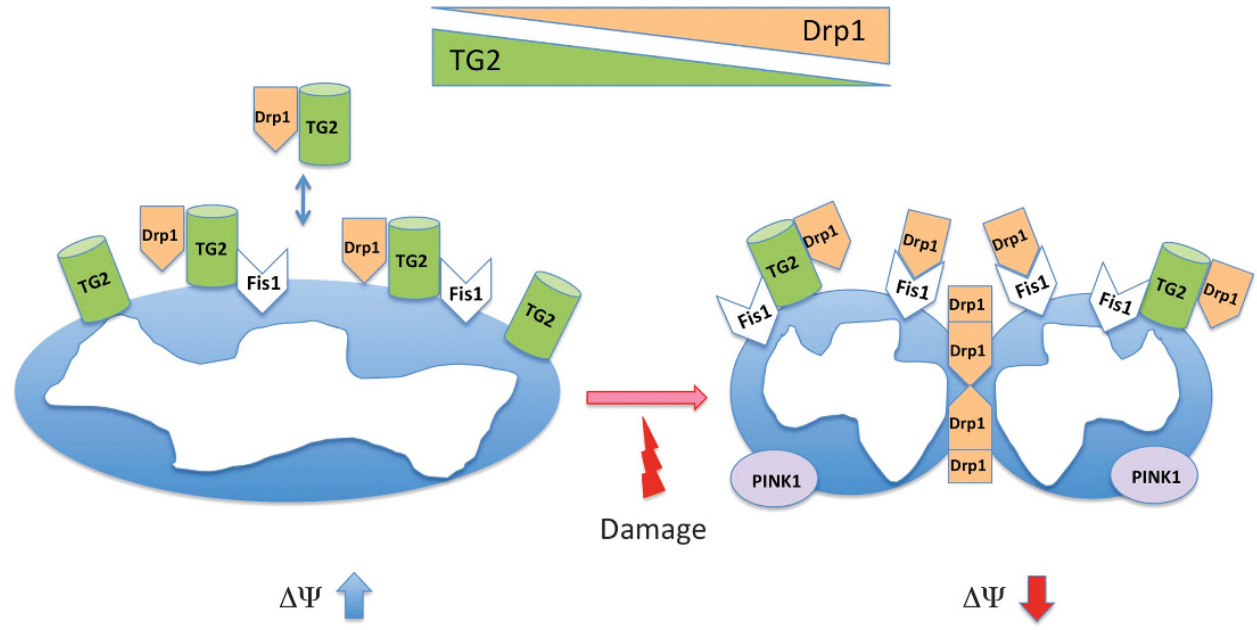

Figure 8 Schematic representation of the hypothetical regulation of mitochondrial dynamics by TG2. In healthy mitochondria TG2 interacts with Drp1 in both the cytoplasm and at the mitochondrial outer membrane level. In addition, TG2 also interacts with Fis1 at the mitochondrial level; this binding could compete for the receptor interaction with its ligand, Drp1. Following mitochondrial damage with CCCP, we observed a significant reduction of the TG2 localized at the mitochondrial level that is paralleled by an almost complete abolishment of its interaction with both Drp1 and Fis1. This event is followed by a much higher Drp1 recruitment on the mitochondria and an increased PINK1 stabilization, thus favouring mitochondrial fragmentation and eventually their removal by mitophagy 
Materials and Methods

Antibodies. The following antibodies were used: anti-Actin (Sigma, St. Louis, MO, USA; A-2066), anti-Tubulin (Sigma, T-4026) anti-Caspase 3 (Cell Signaling, Danvers, MA, USA; 9662), anti-TG2 (Cell Signaling, 3557), anti-VCP (Santa Cruz, Santa Cruz, CA, USA; sc-57492), anti-Tom20 (Santa Cruz, sc-11415), anti-Mitofusin1 (Santa Cruz, sc-50330), anti-GAPDH (Advanced Immuno Chemical, Long Beach, CA, USA; RGM2), anti-Drp1 (BD, San Jose, CA, USA; 611113), anti-IF1 (Abcam, Cambridge, UK; ab-110277), anti-Hsp60 (Enzo SPA-806), anti-Fis1 (AdipoGen, San Diego, CA, USA; AG-25B-0007), anti-HIF-1 $\beta$ (Novus Biologicals, Littleton, CO, USA; NB100-124) and anti-PINK1 (Novus Biologicals, BC100-494). HRP-conjugated secondary antibodies (Bio-Rad Laboratories, Hercules, CA, USA), Alexa Fluor 488-conjugated secondary antibody (Invitrogen, Carlsbad, CA, USA) and Alexa Fluor 594-conjugated secondary antibody (Invitrogen) were used.

Cell culture and drug treatments. MEFs (mouse embryonic fibroblasts), HEK293 (human embryonic kidney) and HEK293 ${ }^{\text {TG2 } 23}$ were cultured in Dulbecco's modified Eagle's medium (Lonza, Basel, Switzerland) supplemented with $10 \%$ fetal bovine serum, $2 \mathrm{mM}$ L-glutamine, $100 \mathrm{mg} / \mathrm{ml}$ streptomycin and 100 units $/ \mathrm{ml}$ penicillin in a $5 \% \mathrm{CO}_{2}$ incubator.

To inhibit autophagy and mitophagy, cells were incubated in full medium for the indicated periods respectively with $20 \mathrm{mM} \mathrm{NH}_{4} \mathrm{Cl}$ (Sigma, A-0171) and $50 \mu \mathrm{M}$ Mdivi-1 (Enzo, BML-CM127). For TG2 inhibition treatment, cells were incubated in full medium in the presence of $40 \mu \mathrm{M}$ Z-DON (Zedira, Darmstadt, Germany) for 18 and $24 \mathrm{~h}$. To induce mitochondrial damage, MEF cells and HEK293 cells were incubated in full medium respectively with 20 and $10 \mu \mathrm{M}$ CCCP (Sigma, C2759). To inhibit glycolysis, cells were incubated in full medium with $10 \mathrm{mM}$ 2-DG (Sigma, D6134).

Immunoblotting. Cells were collected in a lysis buffer containing $20 \mathrm{mM}$ Tris-HCl, pH 7.4, $150 \mathrm{mM} \mathrm{NaCl}$ and $1 \%$ Triton X-100 with protease inhibitor cocktail (Roche, Nutley, NJ, USA). Mitochondria were prepared by differential centrifugation: unbroken cells, nuclei and large membranes were removed through centrifugation at $500 \mathrm{~g}$. The supernatant was further centrifugated at $9000 \times g$. The pellet, which constituted the enriched mitochondrial fraction, was resuspended in $50 \mathrm{mM}$ Tris- $\mathrm{HCl}, \mathrm{pH} 7.4$, and $1 \mathrm{mM}$ EDTA with protease inhibitor cocktail (Roche).

Proteins were quantified with standard Bradford staining and resolved on SDSpolyacrylamide gel and transferred to a nitrocellulose membrane. Blots were blocked in $5 \%$ nonfat dry milk for $1 \mathrm{~h}$ at room temperature and then incubated overnight with the primary antibodies. The membranes were incubated with HRPconjugated secondary antibody for $1 \mathrm{~h}$ at room temperature and the signal was detected by Immun-Star WesternC Kit (Bio-Rad Laboratories).

In situ TG assay. In situ TG activity was quantified by determining the incorporation of BAP into protein substrates. In brief, cells were incubated with 2 mM BAP (Soltec Ventures, Beverly, MA, USA; B110) in combination with each treatment. Cells were then harvested, total extracts or mitochondrial/cytosolic fractions were obtained as above described and proteins were resolved by SDSpolyacrylamide gel. The blots were probed with HRP-conjugated streptavidin (Amersham Biosciences, Little Chalfont, UK) for $2 \mathrm{~h}$ at room temperature and then developed following the standard ECL protocol.

Fluorescence microscopy. MEF cells, grown on coverslips, were incubated with 75 nM Lyso Tracker Red (Molecular Probes, Carlsbad, CA, USA; DND-99) in the culture medium at $37^{\circ} \mathrm{C}$ for 20 min to label acidic compartments, then washed with PBS and fixed in $4 \%$ paraformaldehyde for $10 \mathrm{~min}$ at room temperature. Fixed cells were permeabilized with $0.1 \%$ Triton X-100 in PBS for 10 min, blocked with $3 \%$ BSA in PBS for $20 \mathrm{~min}$ and incubated with anti-Tom20 primary antibody for $1 \mathrm{~h}$. After washing, cells were incubated with Alexa Fluor 488-conjugated secondary antibody. Nuclei were stained with $10 \mu \mathrm{g} / \mathrm{ml}$ Hoechst 33342 for $10 \mathrm{~min}$. The coverslips were mounted on microscope slides, sealed with an antifade solution and examined with an image workstation DeltaVision (Applied Precision, Issaquah, WA, USA) Olympus $1 \times 70$ microscope (Olympus, Munster, Germany).

Immunoprecipitation. Mitochondrial fraction was prepared in HEMG buffer (25 mM Hepes, pH 8.0, $100 \mathrm{mM} \mathrm{NaCl}, 25 \mathrm{mM} \mathrm{MgCl}, 0.5 \%$ Triton X-100, $0.1 \mathrm{mM}$ EDTA and $10 \%$ glycerol) and protease inhibitor cocktail (Roche) as described above. Mitochondrial proteins were subjected to immunoprecipitation using specific antibodies in a combination with Protein G PLUS-Agarose (Santa Cruz). Immunoblot analysis was performed with indicated antibodies.
Quantitative analysis of tubular and fragmented mitochondria. MEF cells were grown on coverslips and then transfected with pDsRed2-Mito plasmid, which leads to the expression of a MitoRed mitochondrial-targeted fluorescent protein, to label the organelles. The transfected cells were fixed in $4 \%$ paraformaldehyde for $10 \mathrm{~min}$ at room temperature and analysed by fluorescence microscopy. Mitochondrial morphology in individual cells was evaluated. Fragmented mitochondria were shortened, punctate and sometimes rounded, whereas tubular mitochondria showed a thread-like tubular structure. For quantification, the cells with different mitochondrial morphologies were counted to determine the percentage of cells with fragmented mitochondria, in five independent fields from three independent experiments. Most of the cells had fragmented mitochondria or tubular mitochondria. If a cell showed mitochondria with mixed morphologies, we classified the mitochondrial morphology according to the majority of the mitochondria.

Measure of glycolytic flux. ECAR was measured using a XF-24 Extracellular Flux Analyzer (Seahorse Bioscience, North Billerica, MA, USA). Primary MEFs were seeded at a density of 60000 cells/well and analysed after $24 \mathrm{~h}$. Optimal cells densities were determined experimentally to ensure a proportional oxygen consumption with cell number in response to FCCP (data not shown). Glycolysis was measured as ECAR that reflects lactate production via glucose catabolism. The experiment was performed in non-buffered DMEM containing $5 \mathrm{mM}$ glucose and $1 \mathrm{mM}$ sodium pyruvate; $\mathrm{pH}$ was adjusted to 7 with $\mathrm{NaOH}$.

Analysis of mitochondrial membrane potential and mitochondrial mass. Determination of mitochondrial membrane potential was performed with tetramethylrodhamine methyl ester (TMRM, Invitrogen). Briefly, WT and KO MEFs were harvested, washed and resuspended in PBS. Cells were then loaded with $200 \mathrm{nM}$ TMRM in PBS for $20 \mathrm{~min}$ at $37^{\circ} \mathrm{C}$.

Mitochondrial mass was measured by MTG (Molecular Probes) staining. Cells were trypsinized, washed and resuspended in PBS with $100 \mathrm{nM}$ MTG for 30 min at $37^{\circ} \mathrm{C}$.

Fluorescence was measured by FACScan flow cytometer (BD Biosciences, San Jose, CA, USA) using the CellQuest software (BD). In each analysis, 10000 events were recorded.

Imaging mitochondrial membrane potential. We used TMRM $(50 \mathrm{nM}$, Invitrogen) in 'redistribution mode': the dye was allowed to equilibrate and was present continuously. TMRM distributes between cellular compartments in response to different potentials and, at concentrations of $<50 \mathrm{nM}$, the fluorescent signal shows a simple relationship with the dye concentration, so that signal intensity maps to mitochondrial potential. TMRM fluorescence intensity was quantified by removing all background signal by 'thresholding' and measuring the mean TMRM fluorescence intensity in the pixels containing mitochondria. Thus, the signal is independent of mitochondrial mass and only reflects the dye concentration within individual mitochondrial structures.

Electron microscopy. WT and KO MEFs were fixed with $2.5 \%$ glutaraldehyde in $0.1 \mathrm{M}$ cacodylate buffer for $1 \mathrm{~h}$ at $4{ }^{\circ} \mathrm{C}$, and postfixed in $1 \%$ osmium tetroxide in $0.1 \mathrm{M}$ cacodylate buffer for $1 \mathrm{~h}$. The cells were then dehydrated in graded ethanol and embedded in Spurr resin. Ultrathin sections were stained with $2 \%$ uranyl acetate and observed under a Zeiss EM900 (Oberkochen, Germany) transmission electron microscope. Images were captured digitally with a Mega View II digital camera (SIS, Olympus).

Statistical analysis. Densitometry measurements were used for statistical analysis using GraphPad (La Jolla, CA, USA). Statistical significance was determined using Student's $t$-test. A $P$-value, ${ }^{*} P<0.05$, was considered to be significant.

\section{Conflict of Interest}

The authors declare no conflict of interest.

Acknowledgements. This work was supported by grants from MIUR (PRIN 2012), Compagnia di San Paolo, the Ministry of Health of Italy 'Ricerca Corrente' and 'Ricerca Finalizzata' and AIRC. The support of the EU grant 'Transpath' Marie 
Curie project to MP is also acknowledged. PGM is supported by a grant from the Netherlands Genomics Initiative (NGI/NWO 05040202), a Marie Curie grant (IRG 247918) and the CEREBRAD grant under the EU-FP7 framework (project number 295552). The Extracellular Flux Analyzer by Seahorse Bioscience was purchased thanks to a generous donation from the 'Dorpmans-Wigmans Stichting' (PGM). The research activities of MC' $s$ laboratory are supported by the BBSRC New Investigator Award Grant (BB//013695/1), the PPCT Research Grant, the Central Research Fund of the University of London, EBRI-Rita Levi Montalcini Foundation Research Programme in Metabolism in Brain Diseases, LAM Research Grant on Brain Tumours, Marie Curie CIG (304165). We thank Dr. P Mattioli from the Centre of Avanced Microscopy, Department of Biology, University of Rome Tor Vergata, for her skillful assistance in the use of the facility.

1. Johansen $T$, Lamark $T$. Selective autophagy mediated by autophagic adapter proteins. Autophagy 2011; 7: 279-296.

2. Monastyrska I, Rieter E, Klionsky DJ, Reggiori F. Multiple roles of the cytoskeleton in autophagy. Biol Rev Camb Philos Soc 2009; 84: 431-448.

3. Mizushima N. Autophagy: process and function. Genes Dev 2007; 21: 2861-2873.

4. Levine B, Klionsky DJ. Development by self-digestion: molecular mechanisms and biological functions of autophagy. Dev Cell 2004; 6: 463-477.

5. Huang J, Klionsky DJ. Autophagy and human disease. Cell Cycle 2007; 6: 1837-1849.

6. Levine B, Kroemer G. Autophagy in the pathogenesis of disease. Cell 2008; 132: 27-42.

7. Sarkar NK, Clarke DD, Waelsh H. An enzymically catalyzed incorporation of amines into proteins. Biochim Biophys Acta 1957; 25: 451-452.

8. Csosz E, Bagossi P, Nagy Z, Dosztanyi Z, Simon I, Fesus L. Substrate preference of transglutaminase 2 revealed by logistic regression analysis and intrinsic disorder examination. J Mol Biol 2008; 383: 390-402.

9. Facchiano $A$, Facchiano $F$. Transglutaminases and their substrates in biology and human diseases: 50 years of growing. Amino Acids 2009; 36: 599-614.

10. Di Venere A, Rossi A, De Matteis F, Rosato N, Agrò AF, Mei G. Opposite effects of $\mathrm{Ca}(2+)$ and GTP binding on tissue transglutaminase tertiary structure. J Biol Chem 2000; 275: 3915-3921.

11. Gundemir S, Colak G, Feola J, Blouin R, Johnson GV. Transglutaminase 2 facilitates or ameliorates HIF signaling and ischemic cell death depending on its conformation and localization. Biochim Biophys Acta 2013; 1833: 1-10.

12. Nakaoka H, Perez DM, Baek KJ, Das T, Husain A, Misono $K$ et al. Gh: a GTP-binding protein with transglutaminase activity and receptor signaling function. Science 1994; 264: 1593-1596.

13. Piacentini M, Farrace MG, Piredda $L$, Matarrese $P$, Ciccosanti $F$, Falasca $L$ et al. Transglutaminase overexpression sensitizes neuronal cell lines to apoptosis by increasing mitochondrial membrane potential and cellular oxidative stress. J Neurochem 2002; 81: 1061-1072.

14. Malorni W, Farrace MG, Matarrese P, Tinari A, Ciarlo L, Mousavi-Shafaei $P$ et al. The adenine nucleotide translocator 1 acts as a type 2 transglutaminase substrate: implications for mitochondrial-dependent apoptosis. Cell Death Differ 2009; 16: 1480-1492.

15. Lorand L, Graham RM. Transglutaminases: crosslinking enzymes with pleiotropic functions. Nat Rev Mol Cell Biol 2003; 4: 140-156.

16. Zemskov EA, Janiak A, Hang J, Waghray A, Belkin AM. The role of tissue transglutaminase in cell-matrix interactions. Front Biosci 2006; 11: 1057-1076.

17. Malorni W, Farrace MG, Rodolfo C, Piacentini M. Type 2 transglutaminase in neurodegenerative diseases: the mitochondrial connection. Curr Pharm Des 2008; 14: 278-288.

18. Gundemir S, Johnson GV. Intracellular localization and conformational state of transglutaminase 2: implications for cell death. PLoS One 2009; 4: 6123.

19. Park D, Choi SS, Ha KS. Transglutaminase 2: a multi-functional protein in multiple subcellular compartments. Amino Acids 2010; 39: 619-631.

20. Piacentini M, D'Eletto M, Falasca L, Farrace MG, Rodolfo C. Transglutaminase 2 at the crossroads between cell death and survival. Adv Enzymol Relat Areas Mol Biol 2011; 78 : $197-246$.

21. Akar U, Ozpolat B, Mehta K, Fok J, Kondo Y, Lopez-Berestein G. Tissue transglutaminase inhibits autophagy in pancreatic cancer cells. Mol Cancer Res 2007; 5: 241-249.

22. D'Eletto M, Farrace MG, Falasca L, Reali V, Oliverio S, Melino G et al. Transglutaminase 2 is involved in autophagosome maturation. Autophagy 2009; 5: 1145-1154.

23. D'Eletto M, Farrace MG, Rossin F, Strappazzon F, Giacomo GD, Cecconi F et al. Type 2 transglutaminase is involved in the autophagy-dependent clearance of ubiquitinated proteins. Cell Death Differ 2012; 19: 1228-1238.

24. Rossin F, D'Eletto M, Macdonald D, Farrace MG, Piacentini M. TG2 transamidating activity acts as a reostat controlling the interplay between apoptosis and autophagy. Amino Acids 2012; 42: 1793-1802

25. Battaglia G, Farrace MG, Mastroberardino PG, Viti I, Fimia GM, Van Beeumen J et al. Transglutaminase 2 ablation leads to defective function of mitochondrial respiratory complex I affecting neuronal vulnerability in experimental models of extrapyramidal disorders. J Neurochem 2007; 100: 36-49.

26. Mastroberardino PG, Farrace MG, Viti I, Pavone F, Fimia GM et al. "Tissue" transglutaminase contributes to the formation of disulphide bridges in proteins of mitochondrial respiratory complexes. Biochim Biophys Acta 2006; 1757: 1357-1365.

27. Rodriguez-Enriquez S, Kim I, Currin RT, Lemasters JJ. Tracker dyes to probe mitochondrial autophagy (mitophagy) in rat hepatocytes. Autophagy 2006; 2: 39-46.

28. Gegg ME, Cooper JM, Chau KY, Rojo M, Schapira AH, Taanman JW. Mitofusin 1 and mitofusin 2 are ubiquitinated in a PINK1/parkin-dependent manner upon induction of mitophagy. Hum Mol Genet 2010; 19: 4861-4870.

29. Ziviani E, Whitworth AJ. How could Parkin-mediated ubiquitination of mitofusin promote mitophagy? Autophagy 2010; 6: 660-662.

30. Springer W, Kahle PJ. Regulation of PINK1-Parkin-mediated mitophagy. Autophagy 2011; 7: 266-278.

31. Rimessi A, Bonora M, Marchi S, Patergnani S, Marobbio CM, Lasorsa FM et al. Perturbed mitochondrial $\mathrm{Ca} 2+$ signals as causes or consequences of mitophagy induction. Autophagy 2013; 9: 1677-1686.

32. Zhang C, Yuan XR, Li HY, Zhao ZJ, Liao YW, Wang XY et al. Downregualtion of dynamin-related protein 1 attenuates glutamate-induced excitotoxicity via regulating mitochondrial function in a calcium dependent manner in HT22 cells. Biochem Biophys 2014; 443: 138-143

33. Chen H, Chan DC. Mitochondrial dynamics - fusion, fission, movement, and mitophagy - in neurodegenerative diseases. Hum Mol Genet 2009; 18: R169-R176.

34. Pi H, Xu S, Zhang L, Guo P, Li Y, Xie J et al. Dynamin 1-like-dependent mitochondrial fission initiates overactive mitophagy in the hepatotoxicity of cadmium. Autophagy 2013; 9 : 1780-1800.

35. Twig G, Hyde B, Shirihai OS. Mitochondrial fusion, fission and autophagy as a quality control axis: the bioenergetic view. Biochim Biophys Acta 2008; 1777: 1092-1097.

36. Campanella M, Casswell E, Chong S, Farah Z, Wieckowski MR, Abramov AY et al. Regulation of mitochondrial structure and function by the F1Fo-ATPase inhibitor protein, IF1. Cell Metab 2008; 8: 13-25.

37. Faccenda D, Tan CH, Duchen MR, Campanella M. Mitochondrial IF1 preserves cristae structure to limit apoptotic cell death signaling. Cell Cycle 2013; 12: 2530-1532.

38. Lefebvre V, Du Q, Baird S, Ng AC, Nascimento M, Campanella M et al. Genome-wide RNAi screen identifies ATPase inhibitory factor 1 (ATPIF1) as essential for PARK2 recruitment and mitophagy. Autophagy 2013; 9: 1770-1779.

39. Tarze A, Deniaud A, Le Bras M, Maillier E, Molle D, Larochette $\mathrm{N}$ et al. GAPDH, a novel regulator of the pro-apoptotic mitochondrial membrane permeabilization. Oncogene 2007; 26: $2606-2620$

40. Kathiria AS, Butcher LD, Feagins LA, Souza RF, Boland CR, Theiss AL. Prohibitin 1 modulates mitochondrial stress-related autophagy in human colonic epithelial cells. PLoS One 2012; 7: e31231.

41. Kim NC, Tresse E, Kolaitis RM, Molliex A, Thomas RE, Alami NH et al. VCP is essential for mitochondrial quality control by PINK1/Parkin and this function is impaired by VCP mutations. Neuron 2013; 78: 65-80.

42. Orru S, Ruoppolo M, Francese S, Vitagliano L, Marino G, Esposito C. Identification of tissue transglutaminase-reactive lysine residues in glyceraldehyde-3-phosphate dehydrogenase. Protein Sci 2002; 11: 137-146.

43. Filiano AJ, Bailey CD, Tucholski J, Gundemir S, Johnson GV. Transglutaminase 2 protects against ischemic insult, interacts with HIF1beta, and attenuates HIF1 signaling. FASEB $J$ 2008; 22: 2662-2675.

44. Frank S, Gaume B, Bergmann-Leitner ES, Leitner WW, Robert EG, Catez F et al. The role of dynamin-related protein 1, a mediator of mitochondrial fission, in apoptosis. Dev Cell 2001; 1: 515-525

45. Lackner LL, Nunnari JM. The molecular mechanism and cellular functions of mitochondrial division. Biochim Biophys Acta 2009; 1792: 1138-1144.

46. Ong SB, Subrayan S, Lim SY, Yellon DM, Davidson SM, Hausenloy DJ. Inhibiting mitochondrial fission protects the heart against ischemia/reperfusion injury. Circulation 2010; 121: 2012-2022

47. Tanaka A, Youle RJ. A chemical inhibitor of DRP1 uncouples mitochondrial fission and apoptosis. Mol Cell 2008; 29: 409-410.

48. Cassidy-Stone A, Chipuk JE, Ingerman E, Song C, Yoo C, Kuwana T et al. Chemical inhibition of the mitochondrial division dynamin reveals its role in Bax/Bak-dependent mitochondrial outer membrane permeabilization. Dev Cell 2008; 14: 193-204.

49. Park SW, Kim KY, Lindsey JD, Dai Y, Heo H, Nguyen DH et al. A selective inhibitor of drp1, mdivi-1, increases retinal ganglion cell survival in acute ischemic mouse retina. Invest Ophthalmol Vis Sci 2011; 52: 2837-2843.

50. Strnad P, Zatloukal K, Stumptner C, Kulaksiz H, Denk H. Mallory-Denk-bodies: lessons from keratin-containing hepatic inclusion bodies. Biochim Biophys Acta 2008; 1782 764-774.

51. Høyer-Hansen M, Jäättelä M. Connecting endoplasmic reticulum stress to autophagy by unfolded protein response and calcium. Cell Death Differ 2007; 14: 1576-1582. 